\title{
Degré d'infestation des adventices dans le bassin cotonnier du Sénégal
}

\author{
Ndongo DIOUF ${ }^{1,2 *}$, Mame Samba MBAYE ${ }^{1}$, Moustapha GUEYE ${ }^{2,3,4}$, Jules DIOUF ${ }^{1}$, \\ Samba Laha KA ${ }^{1,3}$, Moussou Kéba CISSOKO ${ }^{3}$, Djibril DIOP ${ }^{1}$, Modou Fall GUEYE ${ }^{1}$, \\ Birane DIENG ${ }^{1}$, Abdou Aziz CAMARA ${ }^{1}$ et Kandioura NOBA ${ }^{1}$
}
${ }^{1}$ Laboratoire de Botanique Biodiversité, Département de Biologie Végétale, Faculté des sciences et technique, Université cheikh Anta Diop, B.P. 5005 Dakar- Fann, Sénégal.
${ }^{2}$ Centre de recherches zootechniques de Kolda, Institut Sénégalais de Recherche Agricoles, B.P 53 Kolda Sénégal.
${ }^{3}$ Centre National de Recherches Agronomiques de Bambey, Institut Sénégalais de Recherche Agricoles, B.P 53 Bambey, Sénégal.
${ }^{4}$ Centre de Recherches Agricole de Saint Louis, Institut Sénégalais de Recherche Agricoles, B.P 240 Saint Louis, Sénégal.
*Auteur correspondant ; E-mail : ndongodiouf87@gmail.com; Tel : +221 774566580

\section{RESUME}

Au Sénégal, les adventices constituent une des contraintes majeures à l'augmentation de la production agricole. Une étude dans l'objectif de caractériser l'enherbement dans les parcelles cotonnières au Sénégal a été réalisée. Celle-ci a été réalisée grâce à la méthode appelée « tour de champs ». A partir de 139 relevés effectués, 204 espèces réparties en 118 genres appartenant à 35 familles botaniques ont été répertoriées. Les Familles des Fabaceae (22,1\%), Poaceae (20,1\%), Malvaceae (9,3\%) et des Cyperaceae (7,8\%) dominent cette flore et représentent près de $60 \%$ de l'effectif total. Les Dicotylédones sont dominantes avec $68,6 \%$. Sur le plan agronomique, Hyptis suaveolens, Pennisetum pedicellatum, Digitaria horizontalis, Dactyloctenium aegyptium et Kyllinga squamulata constituent les adventices majeures. L'analyse de l'indice partiel de nuisibilité révèle que 47 espèces sont potentiellement nuisibles dont dix constituent les plus nuisibles aux cotonniers dans le bassin cotonnier du Sénégal. Une gestion efficace contre ces espèces devrait être envisagée pour réduire les pertes de rendement dues aux adventices.

(C) 2020 International Formulae Group. All rights reserved.

Mots clés : Adventices, nuisibilité, infestation, cotonnier, rendement, Sénégal.

\section{Degree of weed infestation in Senegal's cotton basin}

\begin{abstract}
In Senegal, weeds are one of the major constraints to increasing agricultural production. A study was carried out to characterize grass cover in cotton plots in Senegal. This was carried out using the method known as "tour de champs". Based on 139 surveys, 204 species in 118 genera belonging to 35 botanical families were identified. The Families Fabaceae (22.1\%), Poaceae (20.1\%), Malvaceae (9.3\%) and Cyperaceae (7.8\%) dominate this flora and represent nearly $60 \%$ of the total number of species. Dicotyledons are dominant with 68.6\%. Agronomically, Hyptis suaveonlens, Pennisetum pedicellatum, Digitaria horizontalis, Dactyloctenium
\end{abstract}


aegyptium and Kyllinga squamulata are the major weeds. Analysis of the partial harmfulness index reveals that 47 species are potentially harmful, ten of which are the most harmful to cotton plants in the cotton basin of Senegal. Effective management against these species should be considered to reduce yield losses due to weeds. (c) 2020 International Formulae Group. All rights reserved.

Keywords. Weeds, pests, infestation, cotton, yield, Senegal.

\section{INTRODUCTION}

Au Sénégal, le coton est la deuxième culture de rente après l'arachide. Sa culture concerne 2 millions de producteurs et 15 millions de personnes parmi les plus pauvres (CEDEAO-CSAO/OCDE, 2006). Au Sénégal, il est l'une des principales sources de revenu des populations notamment au Sénégal oriental et en Haute Casamance.

La production agricole au Sénégal surtout celle cotonnière connait une faiblesse considérable. Cette situation est due à plusieurs facteurs dont les plus importants sont le changement climatique (Ndour, 2017) et les adventices (Noba, 2002; Bassène, 2013 ; Mbaye, 2013). Selon Marnotte (1995), dans les régions cotonnières, les adventices constituent des dommages aux rendements agricoles et engendrent des baisses de récolte de 35 à 90\%. Ainsi, pour réduire les contraintes liées à la production de coton, une étude de la flore adventice afin de proposer un itinéraire technique de désherbage est nécessaire. Au Sénégal, les études réalisées sur les adventices du cotonnier se limitent essentiellement à l'étude du peuplement globale (Diouf et al. 2019). Toutefois, la connaissance de la contribution spécifique des adventices s'est avérée très déterminante dans la proposition de méthode de gestion des adventices (Kazi et al., 2010 ; Touré, 2010 ; Ouattara et al., 2016). Cette étude contribue à une meilleure connaissance de la flore et de la végétation des adventices en déterminant leur degré d'infestation et leur nuisibilité dans les parcelles de coton.

\section{MATERIEL ET METHODES \\ La zone d'étude}

La présente étude a été réalisée en Haute Casamance et au Sénégal Oriental qui correspondent aux zones essentielles de production de coton au Sénégal (Figure 1).
Ces zones constituent essentiellement le bassin cotonnier du Sénégal. Il est principalement constitué de sols ferrugineux tropicaux lessivés qui abritent l'essentiel des cultures de coton et de sols gravionnaires (Badiane et Ndour, 2017).

Dans cette zone, la pluviométrie est variable selon les 3 zones climatiques qui constituent le bassin cotonnier du Sénégal (zone sèche, zone médiane et la zone humide). Ainsi, la zone humide qui représente la plus pluvieuse, la pluviométrie moyenne annuelle varie entre 900 à $1200 \mathrm{~mm}$, de 800 à $900 \mathrm{~mm}$ dans la zone médiane. La zone sèche reçoit en moyenne entre 700 à $800 \mathrm{~mm}$ par an (Dacosta et Kandia, 2002).

\section{Matériel d'étude}

La variété de coton utilisée est celle cultivée au Sénégal, STAM 129 A, elle est originaire du Togo et vulgarisée en 2014. La flore adventice inventoriée est constituée des adventices naturellement émergées dans les parcelles de coton. Des fiches de relevés phytosociologiques ont été utilisées pour répertorier les adventices.

\section{Méthode d'étude}

Des relevés phytosociologiques ont été réalisés dans les parcelles expérimentales des stations de l'ISRA, dans les antennes multi locales d'expérimentation de la SODEFITEX et dans les parcelles paysannes. La technique de relevé floristique qui a été utilisée est celle du «tour de champs», qui consiste à parcourir la parcelle dans différentes directions (Maillet, 1981).

Pour la nomenclature des espèces, la flore du Sénégal (1967) et le Lebrun et Stork (1991-1997) ont été utilisés. Lors du tour de champs, l'indice d'abondance/dominance de chaque adventice a été estimé à partir de l'échelle de Braun-Blanquet (1952) : 
1 = individus peu abondants ou abondants, mais à recouvrement faible ;

$2=$ individus très abondants ou recouvrant $1 / 20$ de la surface ;

3 = individus recouvrant de $1 / 4$ à $1 / 2$ de la surface, abondance quelconque ;

4 = individus recouvrant de $1 / 2$ à $3 / 4$ de la surface, abondance quelconque ;

$5=$ individus recouvrant plus des $3 / 4$ de la surface, abondance quelconque.

L'analyse quantitative de la flore a permis de définir l'importance agronomique des différentes espèces en tenant compte de leur fréquence relative et de leur abondance/dominance moyenne (Le Bourgeois et Guillerm, 1995 ; Touré, 2010) (Tableau 1).
Pour évaluer la nuisibilité des adventices sur les cotonniers, l'indice de l'abondance-dominance et la fréquence absolue ont été notés pour chaque espèce. Ces paramètres ont permis d'attribuer à chaque espèce un indice partiel de nuisibilité (I.P.N.) (Bouhache et al., 1984; Zidane, 2010). Celui-ci s'obtient en transformant l'indice d'abondance-dominance en pourcentage de recouvrement moyen. La combinaison de l'abondance moyen et de la fréquence absolue des espèces donne une idée sur les risques potentiels à l'échelle régionale (Barralis, 1976 ; Bouhache et al. 1984) (Tableau 1).

En conformité aux travaux de Zidane (2010), seules les espèces ayant une fréquence supérieure ou égale à 20 sont considérées.

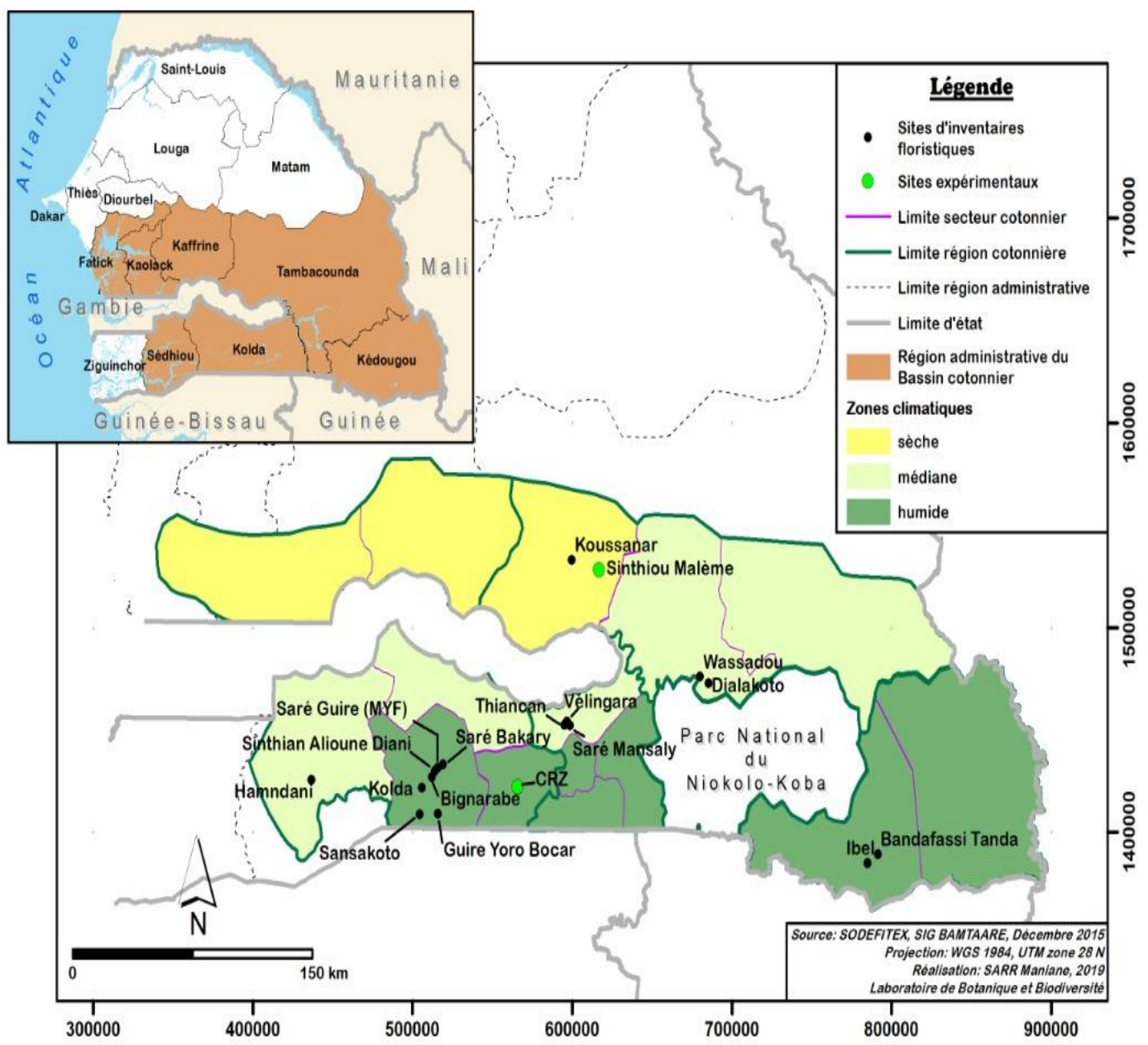

Figure 1 : Carte du bassin cotonnier du Sénégal. 
Tableau 1 : Transformation de l'abondance en pourcentage de recouvrement moyen (Gounot, 1969).

\begin{tabular}{|c|c|c|}
\hline Indice d'abondance-dominance & Classe de recouvrement & Recouvrement moyen en \% \\
\hline 5 & $75-100$ & 87,5 \\
\hline 4 & $50-75$ & 67,5 \\
\hline 3 & $25-50$ & 37,5 \\
\hline 2 & $10-25$ & 17,5 \\
\hline 1 & $1-10$ & 5,5 \\
\hline+ & $0-1$ & 0,5 \\
\hline
\end{tabular}

\section{RESULTATS \\ Richesse floristique}

Sur les 139 relevés réalisés, 204 espèces d'adventices ont été répertoriées en association avec le cotonnier. Ces espèces sont réparties dans 118 genres et 35 familles. Dans cette flore, les dicotylédones dominent avec respectivement $84,8 \%$ des familles, $70,4 \%$ des genres et $69,5 \%$ des espèces (Tableau 2).

Quatre familles dominent nettement la flore adventice dans ce bassin cotonnier : Il s'agit des Fabaceae avec 22,1\%, des Poaceae avec $20,1 \%$, des Malvaceae avec $9,3 \%$ et des Cyperaceae avec $7,8 \%$. Ces familles totalisent à elles seules 117 espèces soit $59,4 \%$ de l'effectif global.

\section{Degré d'infestation}

L'analyse du diagramme d'infestation montre l'existence de 9 groupes d'espèces sur 9 reflétant leur potentiel d'infestation et donc leur importance agronomique.

L'analyse du diagramme d'infestation des espèces recensées dans les cultures cotonnières montre que les espèces sont différemment réparties dans les parcelles. En effet, 9 groupes d'espèces sont mis en évidence (Figure 2).

- Le groupe $1(G 1)$ rassemble les mauvaises herbes majeures générales. Ce sont les espèces les plus infestantes. Ce groupe est représenté par 5 espèces : Hyptis suaveolens, Digitaria horizontalis, Pennisetum pedicellatum, Kyllinga squamulata et Dactyloctenium aegyptium. Ces espèces sont rencontrées dans presque toutes les parcelles avec une abondancedominance supérieur à 1,5 . Leur grande capacité d'adaptation à l'environnement leur confère un potentiel d'envahissement important des parcelles.

- Le groupe 2 (G2) renferme les espèces potentielles générales. Ce groupe est représenté par 6 espèces à savoir Mitracarpus villosus, Commelina benghalensis, Cenchrus biflorus, Fimbristylis hispidula, Spermacoce stachydae et Ipomoae eriocarpa.

- Le groupe 3 (G3), représenté par Hibiscus asper, Cassia obtusifolia, Alysicarpus ovalifolius, Cynodon dactylon et Abelmochus esculentus. Elles sont très ubiquistes mais ne posent pas de problèmes majeurs à la culture. Ces espèces sont présentes dans presque tous les relevés avec une abondance dominance moyenne inférieure à 1,25 et une fréquence relative supérieure à $50 \%$. Elles constituent les mauvaises herbes générales. Toutefois, ces espèces sont à surveiller du fait de leur grande distribution dans les parcelles.

- Le groupe 4 (G4) renferme les espèces majeures régionales telles que Brachiaria stigmatisata, Ipomoae heterotricha. Achyrantes aspera... Ces espèces sont présentes dans 20 à $50 \%$ des parcelles avec un recouvrement très élevés dans le bassin cotonnier du Sénégal.

- Le groupe 5 (G5) dans lequel on retrouve des adventices telles que Euphorbia heterophylla, Eragrostis ciliaris qui 
appartiennent au groupe des mauvaises herbes potentielles régionales.

- Le groupe 6 (G6) compte les adventices dites régionales. On note dans ce groupe Ageratum conysoides, Celosia trygina, Cassia nigricans. Ces espèces ont un spectre écologique large ou moyen. Elles peuvent servir d'espèces indicatrices de région.

- Le groupe $7(\boldsymbol{G} 7)$ rassemble les adventices majeures locales. Ces espèces se caractérisent par une amplitude écologique très étroite. Dans ce groupe, les espèces comme Cyperus esculentus Paspalum orbiculare et Ludwisia hyssopifolia sont notées. Ces espèces sont rencontrées dans peu de relevés mais elles sont très abondantes dans leur milieu.

- Le groupe $8(G 8)$ constitué par les espèces potentielles locales. Ces espèces ont été identifiées dans quelques parcelles dans lesquelles elles se sont révélées abondantes. Dans ce groupe on peut citer entre autres Blumea aurita, Paspalum orbiculare, Ipomoea vagans...

- Le groupe 9 (G9) comprend les adventices mineurs. Elles sont peu fréquentes et peu abondantes et ne constituent pas de contraintes pour la culture de coton au Sénégal. Ce sont donc des espèces non nuisibles et représentent numériquement le groupe le plus important de la flore adventice dans le bassin cotonnier du Sénégal. On peut citer entre autres Indigofera macrocalyx, Cleome viscosa, Oplismenus birmanii, Phyllantus amarus, Scoparia dulcis ect

Tableau 2 : Richesse spécifique des familles et leur proportion.

\begin{tabular}{lcc}
\hline Famille & Nombre d'espèces & Proportion (\%) \\
\hline Fabaceae (D) & 45 & 22,1 \\
Poaceae (M) & 41 & 20,1 \\
Malvaceae (D) & 19 & 9,3 \\
Cyperaceae (M) & 16 & 7,8 \\
\hline
\end{tabular}

\section{Indice partiel de nuisibilité}

Le classement des adventices selon leur indice partiel de nuisibilité et leur fréquence relative a révélé l'existence de 47 espèces pouvant être nuisibles aux cultures cotonnières au Sénégal. Ces espèces sont réparties dans 3 groupes distincts (Tableau 3).

Par référence aux catégories d'adventices définies par Zidane (2010), l'analyse des résultats montre que les adventices dans le bassin cotonnier sont diversement réparties en fonction de leur nuisibilité. Ainsi, trois catégories sont distinguées (Tableau 3) :

IPN $\geq 1000$ : Ce groupe représente les espèces les plus nuisibles dans les parcelles de coton et renferme une dizaine d'espèces telles que Mitacarpus villosus, Hyptis suaveolensis, Digitaria horizontalis, Pennisetum pedicellataum, Dactylovtenuim aegyptium, Spermacoce stachydae, Kyllinga squamulata, Fimbrystilis hispidula, Commelina benghalensis et Ipomoae eriocarpa.

$500 \leq \mathrm{IPN} \leq 1000$ : Dans ce groupe, les espèces présentent une nuisibilité moins prononcée que celle précédemment citée. Toutefois, elles sont fréquentes dans toutes les parcelles avec un recouvrement important. $\mathrm{Ce}$ groupe est le moins diversifié et n'est représenté que par 6 espèces.

IPN $\leq 500$ : ce groupe représente le plus diversifié des catégories d'adventices nuisibles dans le bassin cotonnier du Sénégal avec 31 espèces. Dans ce groupe, on note les espèces présentes avec des recouvrements faibles. Toutefois, elles peuvent êtres très fréquentes dans les parcelles. Parmi celle-ci on peut citer Chloris pilosa, Sesbania pachycarpa, Cyperus rotundus... 


\begin{tabular}{|c|c|c|}
\hline Convolvulaceae (D) & 10 & 4,9 \\
\hline Asteraceae (D) & 10 & 4,9 \\
\hline Rubiaceae (D) & 10 & 4,9 \\
\hline Cucurbitaceae (D) & 6 & 2,9 \\
\hline Amaranthaceae (D) & 4 & 2,0 \\
\hline Combreataceae (D) & 4 & 2,0 \\
\hline Euphorbiaceae (D) & 4 & 2,0 \\
\hline Commelinaceae (M) & 3 & 1,5 \\
\hline Lamiaceae (D) & 3 & 1,5 \\
\hline Acantaceae (D) & 2 & 1,0 \\
\hline Araceae (D) & 2 & 1,0 \\
\hline Nyctaginaceae (D) & 2 & 1,0 \\
\hline Pedaliaceae (D) & 2 & 1,0 \\
\hline Solanaceae (D) & 2 & 1,0 \\
\hline Vitaceae (D) & 2 & 1,0 \\
\hline Aizoaceae (D) & 2 & 1,0 \\
\hline Asparagaceae (M) & 1 & 0,5 \\
\hline Capparaceae (D) & 1 & 0,5 \\
\hline Caryophyllaceae (D) & 1 & 0,5 \\
\hline Colchicaceae (D) & 1 & 0,5 \\
\hline Icacinaceae (D) & 1 & 0,5 \\
\hline Liliaceae (M) & 1 & 0,5 \\
\hline Loganiaceae (D) & 1 & 0,5 \\
\hline Onagraceae (D) & 1 & 0,5 \\
\hline Phyllanthaceae (D) & 1 & 0,5 \\
\hline Portulacaceae (D) & 1 & 0,5 \\
\hline Scrophyllariaceae (D) & 1 & 0,5 \\
\hline Taccaceae (M) & 1 & 0,5 \\
\hline Urticaceae (D) & 1 & 0,5 \\
\hline Verbenaceae (D) & 1 & 0,5 \\
\hline Zygophyllaceae(D) & 1 & 0,5 \\
\hline Total & 204 & 100,0 \\
\hline
\end{tabular}




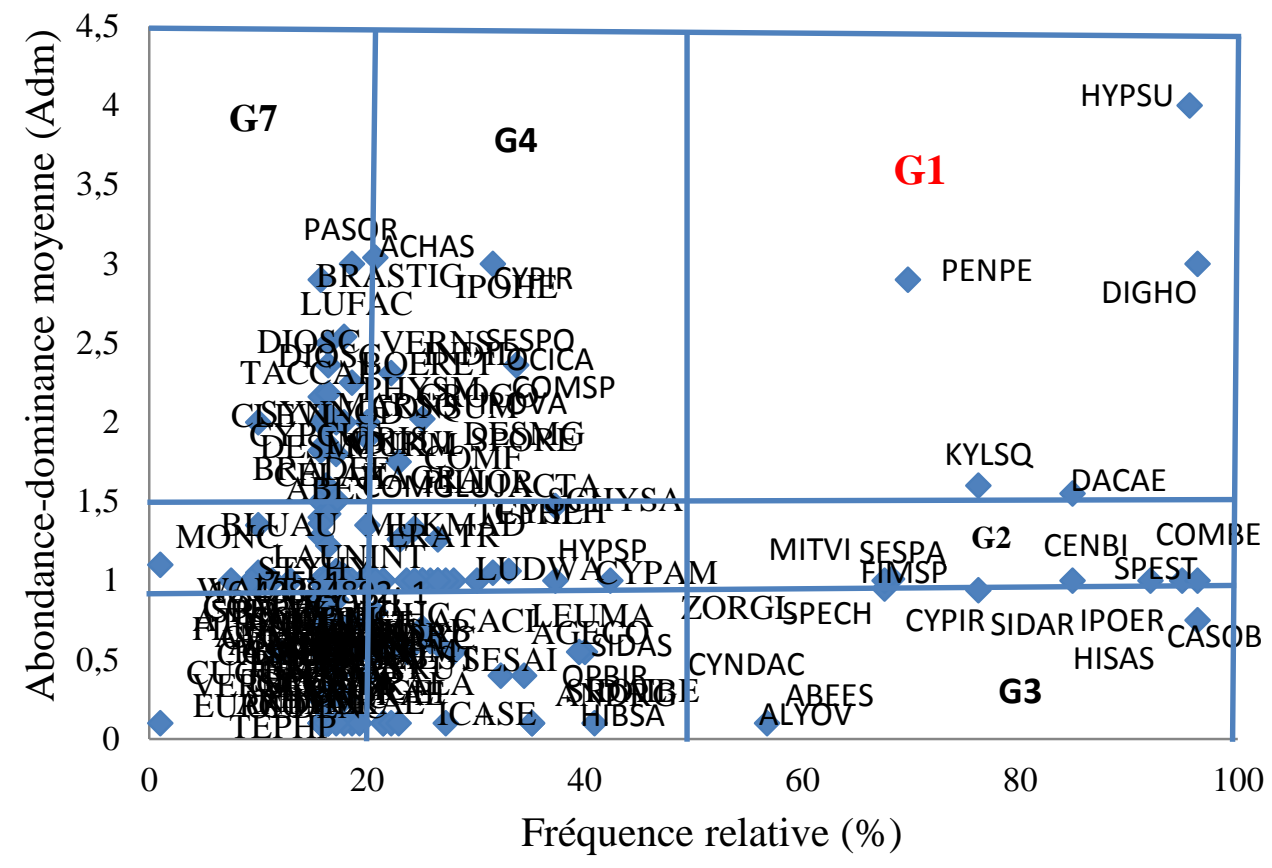

Figure 2 : Diagramme d'infestation des adventices dans le bassin cotonnier du Sénégal.

Tableau 3 : Indice partiel de nuisibilité des adventices répertoriés dans le bassin cotonnier.

\begin{tabular}{lll}
\hline ESPECE & IPN & CATEGORIE \\
\hline Mitracarpus villosus & 2126,3 \\
Hyptus suaveolens & 2117,3 \\
Digitaria horizontalis & 2091,3 \\
Pennisetum pedicellataum & 2005,7 \\
Dactylovtenuim aegyptium & 1485,1 & \\
Spermacoce stachydae & 1326,1 & \\
Kyllinga squamulata & 1270,6 & \\
& & \\
Fimbrystilis hispidula & 1194,7 & \\
Commelina benghalensis & 1065,5 & \\
Ipomoae eriocarpa & 1060,5 & \\
\hline Hibiscus asper & 933,0 & \\
Cyperus iria & 888,6 & Groupe $\mathbf{2}: \mathbf{5 0 0}<\mathbf{0 0 0}$ \\
Celosia trigyna & 727,5 &
\end{tabular}




\begin{tabular}{|c|c|c|}
\hline Cassia obtusifolia & 708,1 & \\
\hline Vernonia galamensis & 684,3 & \\
\hline Cyperus sphacelatus & 610,8 & \\
\hline Chloris pilosa & 493,3 & \\
\hline Sesbania pachycarpa & 463,3 & \\
\hline Cyperus rotundus & 417,0 & \\
\hline Cyperus amabilis & 417,0 & \\
\hline Cyperus esulentus & 417,0 & \\
\hline Achyrantes aspera & 417,0 & \\
\hline Spermacoce chaetocephalla & 374,5 & \\
\hline Colosyntus vulgarus & 347,5 & \\
\hline Mariscus hamilosus & 347,5 & \\
\hline Sesamum indicum & 333,3 & \\
\hline Piliostigma thionnigii & 303,6 & \\
\hline Echinocloa colona & 300,4 & \\
\hline Tephrosia pedicellata & 300,0 & \\
\hline Pycreus lanceolata & 300,0 & \\
\hline Acanthospermum hispidum & 278,0 & Groupe 3 : I.P.N. $\leq 500$ \\
\hline Corchoris tridens & 245,9 & \\
\hline Cyperus difformis & 235,5 & \\
\hline Eragrostis pilosa & 232,8 & \\
\hline Merremia pinnata & 219,2 & \\
\hline Brachiaria lata & 204,5 & \\
\hline Bulbostylis barbata & 173,8 & \\
\hline Andropogon pseudapricus & 172,0 & \\
\hline Tephrosia platycarpa & 170,0 & \\
\hline Ageratum conyzoides & 147,2 & \\
\hline Pennisetum violaceum & 131,1 & \\
\hline Cynodon dactylon & 124,0 & \\
\hline Eragrostis tremula & 111,8 & \\
\hline Merremia pentaphylla & 111,2 & \\
\hline Cassia occidentalis & 104,3 & \\
\hline Brachiaria xantholeuca & 98,0 & \\
\hline Setaria pumula & 94,4 & \\
\hline
\end{tabular}




\section{DISCUSSION}

Cette étude montre que la flore adventice associée au cotonnier est très diversifiée avec 204 espèces réparties dans 118 genres et 35 familles. Cette richesse floristique importante pourrait être expliquée par le fait que la zone soit très pluvieuse avec en moyenne plus de $900 \mathrm{~mm}$ par an. Kouamé et al. (2017) avaient montré que la forte humidité du sol serait favorable à la germination des graines d'adventices enfouies. Cependant, la flore adventice du cotonnier qui est une culture fertilisée est moins diversifiée que celle du sorgho en Haute Casamance où 232 espèces ont été répertoriées (Ka, 2019). Cette dernière ne reçoit pas de fertilisation. Ces résultats suggèrent l'effet de la fertilisation sur la diversité spécifique. La fertilisation, notamment azotée, a pour objectif d'améliorer la productivité. Toutefois, elle n'est pas sans effets sur la flore adventice. Durant et Kernéis (2015) ont montré que le nombre d'espèces et la diversité dans une prairie de fauche en marais charentais ont progressivement diminué avec les doses de fertilisation. Cette variabilité spécifique en fonction des niveaux de fertilisation a aussi été notée dans les systèmes de culture en France (Guay, 2012). En effet, le nombre d'espèces dans les parcelles à bas niveaux d'intrant est presque deux fois plus important que dans les systèmes conventionnels.

Quatre familles sont dominantes dans cette flore adventice; Il s'agit des Fabaceae, Poaceae, Malvaceae et des Cyperaceae. Cellesci figurent parmi les plus représentées dans les zones de transition forêt et savanes en Côte d'Ivoire (Tiébré et al., 2016). Des tendances similaires ont également été obtenues sur la diversité herbacée dans le parc national de Manda au Tchad (Ndotam et al., 2017).

Les résultats de cette étude montrent que les adventices n'ont pas le même degré de nuisibilité dans le bassin cotonnier du Sénégal. Ainsi, 47espèces réparties dans 4 groupes posent plus de problème à la culture de coton.
La nuisibilité de ces espèces d'adventices pourrait être liée entre autres à leur adaptabilité aux conditions de culture et à leur biologie ect. La plupart de ces espèces sont signalées dans les cultures de maïs et de mil dans le sud du bassin arachidier (Bassène, 2014 ; Mbaye, 2013 et Noba, 2002). La plupart de ces espèces se caractérise par des levées persistantes, des pics de germination étalés durant le cycle du cotonnier et par une production de semence importante. Dans le bassin cotonnier, Hyptis suaveolens est considéré comme étant l'espèce la plus nuisible. Sa nuisibilité pourrait être expliquée par le fait qu'elle ait peu d'ennemie naturel et par l'absence de dormance. Elle se caractérise par une forte production de semences, en moyenne 1000 graines/individus (Bassène, 2008). Noba (2002) avait montré que la nuisibilité de Dactyloctenium aegyptium s'explique par une levée précoce et que Mitracarpus villosus et Kyllinga squamulata présentent deux pics de levé. Digitaria horizontalis est l'une des espèces les plus problématiques à la culture du coton en Afrique (Maisoneuve et Larose, 1986). La nuisibilité de cette espèce a été montrée dans plusieurs cultures et dans plusieurs localités. Au Viét Nam, elle est la plus redoutable adventice dans les cultures de maïs et du riz (Stevoux, 2000). Sa nuisibilité est liée par une capacité de forte production de semences. Le Bourgeois et Marnott (1994) ont montré qu'un individu peut produire jusqu'à 1200 graines. A côté de ces espèces, il faut noter une forte présence de Spermacoce stachydae dans les parcelles de coton. Ces résultats pourraient être assimilés à ceux de Bassène (2014) sur le Spermacoce chaetocephalla dont la germination est étalée sur toute la saison. Leur présence serait tributaire d'une absence de dormance tégumentaire. Selon nos observations, la présence de Spermacoce stachydae et de Fimbristylis hispidula pourrait être expliquée par leur supplantation aux graminées après application des herbicides qui constituent la principale méthode de lutte contre les 
adventices dans la zone. L'infestation des parcelles de coton par les espèces telles que Commelina benghalensis et Ipomoea eriocarpa était déjà signalée au Benin par Gounifio (1988) et Ahanchede (1994). Au Sénégal, Fall et al. (1978) font remarquer que les plants de Commelina sont capables de repousser si une pluie survient huit jours après sarclage. Ahanchede (1994) a montré que l'infestation de Commelina benghalensis pourrait être liée sa capacité de multiplication sexuée et végétative et une forte production de graines.

\section{Conclusion}

Dans le but de réunir des informations permettant d'améliorer les méthodes de gestion des adventices dans les cultures cotonnières au Sénégal, le présent travail s'est proposé de caractériser la flore adventice et d'étudier les groupements d'adventices. La flore adventice des cultures cotonnières au Sénégal est caractérisée par une grande diversité systématique, soit 204 espèces, 118 genres appartenant à 35 familles. Les dicotylédones sont dominantes avec $68,6 \%$ de la flore. Quatre familles dominent nettement l'ensemble de la flore, il s'agit des Fabaceae, Poaceae, Malvaceae et des Cyperaceae. Elles représentent plus de la moitié, soit 59,3\% de l'effectif total des espèces inventoriées. De point de vue agronomique, cette étude a permis de mettre en évidence les adventices les plus problématiques des cultures de coton. Quarante-sept (47) espèces sont considérées comme potentiellement nuisibles dont 10 telles que Mitacarpus villosus, Hyptis suaveolensis, Digitaria horizontalis, Pennisetum pedicellataum, Dactylovtenuim aegyptium, Spermacoce stachydae, Kyllinga squamulata, Fimbrystilis hispidula, Commelina benghalensis et Ipomoae eriocarpa constituent les plus nuisibles au cotonnier.

\section{CONFLIT D'INTERETS}

Les auteurs déclarent qu'ils n'ont aucun conflit d'intérêts.

\section{CONTRIBUTIONS DES AUTEURS}

ND a contribué à la rédaction du protocole, a collecté les données, a contribué à l'analyse et au traitement des données et a rédigé le manuscrit. JD, SLK, MKS, DD et MFG ont contribué à la rédaction du manuscrit. AAC a contribué à l'identification des espèces. $\mathrm{KN}, \mathrm{MSM}$ et MG ont supervisé les travaux et ont contribué à la rédaction du manuscrit.

\section{REMERCIEMENTS}

Les auteurs remercient les autorités de l'Université de Dakar et du Département de Biologie Végétales pour la formation, ainsi que l'ISRA pour la mise en œuvre des moyens matériels et financiers qui ont permis l'aboutissement de ce travail et la SODEFITEX pour la collaboration.

\section{REFERENCES}

Ahanchede A et Gasquez J. 1995. Mauvaises herbes des cultures pluviales au nord-est du Benin. Faculté des sciences Agronomiques. Agriculture et développement., 7: 22-29.

Badiane NY et Ndour A. 2017. Situation de la fertilité des sols en culture cotonnière au Sénégal et Propositions de solutions. $7^{\text {ème }}$ réunion du Programme Régional de Protection Intégrée du Cotonnier en Afrique (PR-PICA) du 16 au 18 Avril Dakar, Sénégal.

Bassène C. 2014. La flore adventice dans les cultures de maïs (Zea mays L.) dans le sud du Bassin Arachidier: structure, nuisibilité et mise au point d'un itinéraire de désherbage; Thèse de Doctorat unique. Université Cheikh Anta DIOP de Dakar, Département de Biologie Végétale. $176 \mathrm{p}$.

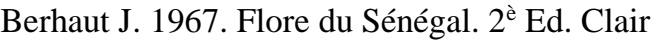
Afrique, Dakar, 257 p. 
Bouhache M. et Boulet C. 1984. Étude floristique des adventices de la tomate dans le Souss. Hommes Terre Eaux, 14(57): 37-49p.

Braun-Blanquet J. 1952. Phytosociologie appliquée. SIGMAT 116: 157-161.

CEDEAO, 2006. Le coton. Rapport CEDEAO. Atlas de l'intégration de l'Afrique de l'Ouest. $20 \mathrm{p}$

Dacosta H., Kandia KY. 2002. La variabilité spatio-temporelle des précipitations au Sénégal depuis un siècle. Conference held at Cape Town. South Africa. March 2002. IAHS Publ. no. 274.2002.

Diouf N, Mbaye MS, Gueye M, Dieng B, Bassène C, Noba K. 2019. Flore adventice des cultures cotonnières dans le Sénégal Oriental et en Haute Casamance. Int. J. Biol. Chem. Sci. 13(3): 1720-1736. DOI:

https://dx.doi.org/10.4314/ijbcs.v13i3.41

Fall M, Hernandez S, Pirot R, Pocthier G, Tchakeriane E. 1978. Utilisation des herbicides au Sénégal: principales contraintes et possibilités d'introduction en milieu paysan. $3^{\text {éme }}$ symposium sur le désherbage des cultures tropicales. Dakar 17 au 21 Septembre. 31- 45p

Gounifio P. 1988 . Biomorphologie de Commelina benghalensis pour une meilleure approche de la lutte raisonnée. Thèse de doctorat. Université des Sciences Techniques du Languedoc. Montpellier $108 \mathrm{p}$

Guay E, 2012- Étude de l'impact sur la flore adventice de plusieurs systèmes de culture à bas niveau d'intrants. Mémoire de Fin d'Etudes. Diplôme d'Ingénieur de l'Institut Supérieur des Sciences Agronomiques, Agroalimentaires, Horticoles et du Paysage. Ecole d'Ingénieurs de Purpan (Toulouse, France). 46 pages + annexes

Ka S L. 2019. Flore adventice du Sorgho (Sorghum bicolor L. Moench) en Haute Casamance : Structure, dynamique, nuisibilité et calendrier de désherbage. Thèse de Doctorat unique de Biologie Végétale. FST, UCAD, Dakar. 169 p.

Kazi Tani Ch, Le Bourgeois T, Munoz F. 2010. Aspects floristiques des adventices du domaine phytogéographique oranais (Nord- Ouest Algérien) et persistance d'espèces rares et endémiques. $\mathrm{Fl}$ Médit. 20 :29-46. ISSN 1120-4052

Kouamé AS, Bakayoko GA, Kouamé KF, Ipou Ipou J, N'guessan KE. 2017. Flore adventice des cultures vivrières de la zone périurbaine du district d'Abidjan (Côte d'Ivoire). Journal of Applied Biosciences 118: 11744-11753 p

Le Bourgeois T, Guillerm JL. 1995. Etendue de distribution et degré d'infestation des adventices dans les rotations cotonnières au Nord-Cameroun. Weed Res. 35(2): 8998

Lebrun JP et Stork AL. 1991 et 1997. Enumération des plantes à fleurs d'Afrique tropicale. Conservatoire et Jardin Botanique de Genève. 4 volumes.

Maillet J. 1981. Evolution de la flore adventice dans le Montpelliérais sous la pression des techniques culturales. Thèse Doctorat, Montpellier, 200p.

Maisoneuve et Larose. 1986. Le coton en Afrique. Sahel point Doc

Marnotte P. 1995. Utilisation des herbicides : contraintes et perspectives. Agriculture et Développement (7): 12-21 p

Mbaye MS. 2013. Association mil [Pennisetum glaucum (L.) R. Br.] et niébé [(Vigna unguiculata (L.) Walp.] : Arrangement spatiotemporel des cultures, structure, dynamique et concurrence de la flore adventice et proposition d'un itinéraire technique. Thèse de Doctorat d'Etat de Biologie Végétale. FST, UCAD, Dakar. 236p.

Ndotam TIN, Reounodji F, Lumandé KJ, Diaouangana J. 2017. Evaluation de la diversité floristique en herbacées dans le 
Parc National de Manda au Tchad. Int. J. Biol. Chem. Sci., 11(4): 1484-1496

Ndour A, Romain L, Gourlot JP, Ba KS, Dieng A, Clouvel P. 2017. Changement climatique et production cotonnière au Sénégal : concevoir autrement les stratégies de diffusion des variétés. SODEFITEX. Bd du Centenaire de la Commune de Dakar, km 4,5. BP 3216. Dakar (Sénégal). Biotechnol. Agron. Soc. Environ. 2017 21(1): 22-35. 14p

Noba K. 2002. La flore adventice dans le sud du Bassin arachidier (Sénégal) : structure dynamique et impact sur la production du mil et de l'arachide. Thèse de Doctorat d'Etat de Biologie Végétale. FST, UCAD, Dakar. 128p.

Ouattara D, Kouamé D, Tiébré MS, Kouadio YJC et N'Guessan KE. 2016. Biodiversité végétale et valeur d'usage en zone soudanienne de la Côte d'Ivoire. Int. J. Biol. Chem. Sci., 10(3): 1122-1138. http://dx.doi.org/10.4314/ijbcs.v10i3.18

Stevoux V. 2000. Caractérisation de l'enherbement des systèmes de cultures traditionnelles de maïs et du riz pluvial dans une zone montagnarde de Viêtnam. Mémoire de master of science. Centre National des Etudes Agronomiques des Régions Chaudes. Montpellier Cedex 01Grance. 120 p.

Tiébré MS, Ouattara D, Kpangui KB, Kouassi DF, N'Guessan KE. 2016. Diversité floristique de la région de Foungbesso en zone de transition forêt - savane à l'ouest de la Côte d'Ivoire. Int. J. Biol. Chem. Sci., 10(3): 1007-1016. DOI : http://dx.doi.org/10.4314/ijbcs.v10i3.8

Touré A., Adou LM. D., Kouamé FK., Ipou J. I. 2016. Dynamique d'infestation de la forêt classée de Sanaimbo par les adventices à partir des agroécosystèmes environnants. Tropicultura, 2016, 34, 4, 361-374p

Zidane L, Salhi S, Fadli M, Antri EM, Taleb A, Douira A. 2010. Etude des groupes d'adventices dans le Maroc occidental. Biotechnol. Agron. Soc. Environ. 2010., 14(1):153-166. 